\title{
On Delay-Fractional-Dependent Stability Criteria for Takagi-Sugeno Fuzzy Systems with Interval Delay
}

\author{
Xianzhong Xia, ${ }^{1}$ Renfa Li, ${ }^{1}$ and Jiyao $\mathrm{An}^{1,2}$ \\ ${ }^{1}$ Key Laboratory of Embedded and Network Computing of Hunan Province, College of Information Science and Engineering, \\ Hunan University, Changsha 410082, China \\ ${ }^{2}$ Department of Applied Mathematics, Faculty of Mathematics, University of Waterloo, Waterloo, ON, \\ Canada N2L 3G1 \\ Correspondence should be addressed to Renfa Li; lirenfa@vip.sina.com
}

Received 21 June 2013; Revised 10 December 2013; Accepted 14 December 2013; Published 17 February 2014

Academic Editor: Mohammed Chadli

Copyright (C) 2014 Xianzhong Xia et al. This is an open access article distributed under the Creative Commons Attribution License, which permits unrestricted use, distribution, and reproduction in any medium, provided the original work is properly cited.

This paper investigates stability program of Takagi-Sugeno fuzzy systems with interval time-varying delay via a variable delay decomposition approach. By developing a delay decomposition approach, both lower and upper bound information of the delayed plant states can be taken into full consideration; two novel delay-fractional-dependent stability criteria are obtained based on the direct Lyapunov method allied with an appropriate and variable Lyapunov-Krasovskii functional choice and with two different bounding techniques to estimate some integral terms in the time-derivative of the Lyapunov-Krasovskii functional. The first stability criterion is derived by utilizing the suitable and generalized integral inequalities, while the second stability condition is obtained by employing a scalar inequality, without any direct approximation. Particularly, the proposed results differ from previous ones since the positiveness of the Lyapunov-Krasovskii functional is guaranteed by new relaxed conditions. When applying these two stability criteria to check the stability of a T-S fuzzy system, it is shown through some numerical examples that the first stability condition can provide a larger maximum allowable delay bound than the second stability criterion, and both stability criteria yield less conservative than the existing ones.

\section{Introduction}

Stability is a central issue in dynamical system and control theory. A dynamical system is called stable (in the sense of Lyapunov) if starting the system somewhere near its desired operating point implies that it will stay around that point ever after [1]. Fuzzy systems are nonlinear systems described by a set of "IF-THEN" rules which give a local linear representation for an underlying system. Consequent function-type fuzzy models with linear consequent functions are called Takagi-Sugeno (T-S) models; using such models, complex nonlinear systems can be represented by selection of membership functions and consequent functions, which can approximate a wide range of nonlinear systems $[2,3]$. In practice, time delays are frequently encountered in many engineering systems, and they are usually regarded as a source of instability and poor performance $[1,3]$. In particular, the problem of checking stability of systems with an interval time-varying delay $\tau(t)$, that is, $\tau(t) \in\left[h_{a}, h_{b}\right]$, is more involving $[4,5]$ and may be found, for example, in networked control systems. As a result, stability analysis for T-S fuzzy systems with interval time-varying delay is of great significance both in theory and in practice. Much effort has been devoted to the analysis and synthesis of fuzzy systems with interval delay during the last decades; for example, see [5-18] and the references therein. Most recently, stability analyses for T-S fuzzy systems also have been extended to the fuzzy control and observer design, $H_{-} / H_{\infty}$ faults detection, and fault tolerant; for example, see [15, 19-23].

Recently, the development of the technologies for delaydependent stability analysis has been focusing on effective reduction of the conservation of the stability conditions. The main aim is to derive a maximum allowable delay bound (MADB) of the time delay such that the time delays' 
system is asymptotically stable for any delay between the lower bound and the MADB. Via different types of LK functional together with the Jensen inequality approach, improved delay-dependent stability results were provided in $[10,12,14,16,17,24,25]$. An et al. $[12,14]$ improved the stability conditions for T-S fuzzy systems with normbounded-type uncertainties by constructing a new LKF and improving Jensen's inequality. Note that most of the aforementioned delay-dependent stability results were obtained by requiring all the involved symmetric matrices in a chosen LK functional to be positive definite. Such a requirement can cause conservatism in the criteria. Actually, this requirement is not necessary to guarantee an LK functional to be positive definite, which is similar to relaxed positiveness condition in [10], though the authors in [10] used a different LK functional. This observation has been overlooked when using the LK functional approach in the literature, which motivates the present study.

In order to reduce the conservatism introduced by model transformation and bounding techniques, a free-weighting matrices method is proposed in $[17,18,26]$. Z. Yang and Y.-P. Yang [18] studied the stability and synthesis problem of uncertain T-S fuzzy systems with time-varying delay by using LKF approach. Liu et al. [26] proposed new delaydependent stability criteria for T-S fuzzy systems with timevarying delay. Recently, a delay partitioning approach has been proposed to further reduce their conservatism; see $[4,9,11-14,27-29]$ and references therein. However, the decomposition approach is complicated and the resulting stability condition is difficult to check in [27]. Liu [28] used a delay decomposition approach, and new stability results were derived in the sense of simpler complexity and less conservatism. Zhao et al. [29] proposed a delay partitioning approach to stability and stabilization of delayed T-S fuzzy systems. Most recently, Peng et al. $[8,9]$ proposed some less conservative results by using delay partitioning technique and free matrices based on Newton-Leibniz formula. Yet, there exists further room to investigate the upper bound of the time-derivative of the LK functional [5-14, 25, 26], which also motivates the present study.

This paper will focus on the delay-fractional-dependent stability problem of T-S fuzzy systems with interval timevarying delay. Inspired by the novel approach in Souza et al. [10], by some integral inequalities in $[1,24,30]$, then by the works of Liu [28], Zhang et al. [24], and Briat [31], and by the aforementioned observation that the positive definiteness of a chosen LK functional does not necessarily require all the involved symmetric matrices to be positive definite, we firstly construct a novel delay-fractional-dependent LK functional by developing a variable delay decomposition technique. Secondly, we estimate the upper bound of its derivative less conservatively by suitably utilizing the integral inequalities and adopt the convex combination technique; then we obtain a less conservative stability criterion in terms of linear matrix inequalities (LMIs). On the other hand, by using a scalar inequality proposed in [32], another stability criterion is also proposed which is less conservative than the existing ones without any free matrices included; that is, there has not any extra computation burden. Finally, three numerical examples are used to compare with some previous results and demonstrate the effectiveness of the proposed method.

\section{Problem Formulation}

Consider a nonlinear system with interval time-varying delay via T-S fuzzy model, which can be described as follows:

Rule j: IF $\theta_{1}(t)$ is $N_{j 1}$ and $\ldots$ and $\theta_{p}(t)$ is $N_{j p}$, THEN

$$
\begin{aligned}
& \dot{x}(t)=A_{j} x(t)+A_{\tau j} x(t-\tau(t)), \\
& x(t)=\phi(t), \quad \forall t \in\left[-h_{b}, 0\right], j=1,2, \ldots, r,
\end{aligned}
$$

where $0 \leq h_{a} \leq \tau(t) \leq h_{b}$ and $x(t)$ is the state vector. $\phi(t)$ is the continuous initial vector function defined on $\left[-h_{b}, 0\right] ; \theta_{1}(t), \theta_{2}(t), \ldots, \theta_{p}(t)$ denote the premise variables while $N_{j 1}, N_{j 2}, \ldots, N_{j p}$ represent the fuzzy sets; the system coefficient matrices are constant real matrices with appropriate dimensions, where $j=1,2, \ldots, r$ and $r$ is the number of IF-THEN rules. For the sake of convenience, we denote $\bar{\tau}=h_{b}-h_{a}$.

In this paper, the delay $\tau(t)$ is assumed to be time-varying delay as the following two cases.

Case I. $\tau(t)$ is a differentiable function, satisfying for all $t \geq 0$

$$
0<h_{a} \leq \tau(t) \leq h_{b}, \quad \dot{\tau}(t) \leq h_{d} .
$$

Case II. $\tau(t)$ is not differentiable or the upper bound of the derivative of $\tau(t)$ is unknown, and $\tau(t)$ satisfies

$$
0<h_{a} \leq \tau(t) \leq h_{b}
$$

where $h_{a}, h_{b}$, and $h_{d}$ are some given values.

The fuzzy system (1) is supposed to have singleton fuzzifier, product inference, and centroid defuzzifier. The final output of the fuzzy system is inferred as follows:

$$
\begin{aligned}
& \dot{x}(t)=A(t) x(t)+A_{\tau}(t) x(t-\tau(t)), \\
& x(t)=\phi(t), \quad \forall t \in\left[-h_{b}, 0\right],
\end{aligned}
$$

where

$$
\begin{gathered}
A(t):=\sum_{j=1}^{r} h_{j}(\theta(t)) A_{j}, \\
A_{\tau}(t):=\sum_{j=1}^{r} h_{j}(\theta(t)) A_{\tau j},
\end{gathered}
$$

and $h_{j}(\theta(t))=\mu_{j}(\theta(t)) / \sum_{i=1}^{r} \mu_{i}(\theta(t)), \mu_{j}(\theta(t))=$ $\prod_{k=1}^{p} N_{j k}\left(\theta_{k}(t)\right)$, and $N_{j k}\left(\theta_{k}(t)\right)$ is the membership function of $\theta_{k}(t)$ in $N_{j k}$. Here $\mu_{j}(\theta(t)) \geq 0$ and $\sum_{j=1}^{r} h_{j}(\theta(t))=1$.

The purpose of this paper is to find new stability criteria, which are less conservative than the existing results. Such a criterion may be used to compute the tolerable delay bound $h_{b}$ for given $h_{a}$ or vice versa. 
To end this section, we introduce the following lemmas which are useful in stability analysis for the T-S fuzzy systems.

First, Lemma 1 induces some integral inequalities.

Lemma 1 (integral inequalities, Gu et al. [1], Zhang et al. [24], and Sun et al. [30]). Let $x(t) \in \mathfrak{R}^{n}$ be a vector-valued function with first-order continuous-derivative entries. Then, for any matrices $M, N \in \mathfrak{R}^{n \times n}, Z \in \mathfrak{R}^{2 n \times 2 n}, X=X^{T} \in \mathfrak{R}^{n \times n}$, and some given scalars $0 \leq \tau_{1}<\tau_{2}$, the following integral inequality holds.

Case 1 . When $X>0$ and $\tau_{1}, \tau_{2}$ are constant values,

$$
\begin{aligned}
& \left(\tau_{2}-\tau_{1}\right) \int_{t-\tau_{2}}^{t-\tau_{1}} x^{T}(s) X x(s) d s \\
& \geq \int_{t-\tau_{2}}^{t-\tau_{1}} x^{T}(s) d s X \int_{t-\tau_{2}}^{t-\tau_{1}} x(s) d s \\
& \left(\frac{\tau_{2}^{2}-\tau_{1}^{2}}{2}\right) \int_{-\tau_{2}}^{-\tau_{1}} \int_{t+\theta}^{t} x^{T}(s) X x(s) d s d \theta \\
& \geq \int_{-\tau_{2}}^{-\tau_{1}} \int_{t+\theta}^{t} x^{T}(s) d s d \theta X \int_{-\tau_{2}}^{-\tau_{1}} \int_{t+\theta}^{t} x(s) d s d \theta, \\
& -\left(\tau_{2}-\tau_{1}\right) \int_{t-\tau_{2}}^{t-\tau_{1}} \dot{x}^{T}(s) X \dot{x}(s) d s \\
& \leq\left[\begin{array}{l}
x\left(t-\tau_{1}\right) \\
x\left(t-\tau_{2}\right)
\end{array}\right]^{T}\left[\begin{array}{cc}
-X & X \\
* & -X
\end{array}\right]\left[\begin{array}{l}
x\left(t-\tau_{1}\right) \\
x\left(t-\tau_{2}\right)
\end{array}\right] .
\end{aligned}
$$

Case 2. When $X>0$ and $\tau_{1}, \tau_{2}$ are time-varying, $h=\tau_{2}-\tau_{1}:=$ $h(t) \geq 0$,

$$
\begin{aligned}
& -\int_{t-\tau_{2}}^{t-\tau_{1}} x^{T}(s) X x(s) d s \\
& \leq\left[\begin{array}{l}
x\left(t-\tau_{1}\right) \\
x\left(t-\tau_{2}\right)
\end{array}\right] \\
& \quad \times\left\{\left[\begin{array}{cc}
M+M^{T} & -M+N^{T} \\
* & -N-N^{T}
\end{array}\right]+h\left[\begin{array}{c}
M \\
N
\end{array}\right] X^{-1}\left[M^{T} N^{T}\right]\right\} \\
& \quad \times\left[\begin{array}{l}
x\left(t-\tau_{1}\right) \\
x\left(t-\tau_{2}\right)
\end{array}\right] .
\end{aligned}
$$

Case 3. When $\tau_{1}, \tau_{2}$ are time-varying, $h=\tau_{2}-\tau_{1}:=h(t) \geq 0$, and $X$ is any symmetric matrix,

$$
\begin{aligned}
& -\int_{t-\tau_{2}}^{t-\tau_{1}} x^{T}(s) X x(s) d s \\
& \leq\left[\begin{array}{l}
x\left(t-\tau_{1}\right) \\
x\left(t-\tau_{2}\right)
\end{array}\right]^{T}\left\{\left[\begin{array}{cc}
M+M^{T} & -M+N^{T} \\
* & -N-N^{T}
\end{array}\right]+h Z\right\} \\
& \quad \times\left[\begin{array}{l}
x\left(t-\tau_{1}\right) \\
x\left(t-\tau_{2}\right)
\end{array}\right]
\end{aligned}
$$

with $\left[\begin{array}{ll}X & Y \\ * & Z\end{array}\right] \geq 0$ and $Y=\left[\begin{array}{ll}M & N\end{array}\right]$.
Remark 2. Equations (6)-(10) are called some integral inequalities presented over the existing literatures. They play a key role in the derivation of a criterion for delay-fractionaldependent stability in this paper. To reduce conservatism of the proposed results, the relaxed matrix inequalities in (6)-(10) will be adopted in the present work. The relaxed matrix inequality is quite different from the ones in [1] and also can produce less conservative results than the descriptor model transformation method [18, 24, 26]. In [610], the Leibniz-Newton formula was employed and many free-weighting matrix variables were introduced. In fact, these free-weighting matrix variables are all redundant and can be eliminated, which was theoretically proven in [16]. Generally speaking, by using Jensen's inequality or equivalent Jensen inequality [31], the computational complexity of the obtained stability criterion may be lower than the ones in [6-10], and thus the integral inequality based results are carried out more efficiently as compared with the general free-weighting matrix based on the Newton-Leibniz formula, since fewer matrix variables are introduced for computing.

Second, we introduce the following scalar inequality which is essential in the proofs of our results without any free matrices.

Lemma 3 (scalar inequality, [32]). For any scales $M \geq 0, N \geq$ 0 , and $h_{2} \leq \tau(t) \leq h_{3}$, one has

$$
\begin{aligned}
& -\frac{h_{3}-h_{2}}{\tau(t)-h_{2}} M-\frac{h_{3}-h_{2}}{h_{3}-\tau(t)} N \\
& \quad \leq \max \{-(M+3 N),-(N+3 M)\} .
\end{aligned}
$$

\section{Main Results}

This section aims to develop a novel delay decomposing approach for stability analysis of T-S fuzzy system (4).

Firstly, we divide the delay intervals $\left[0, h_{a}\right]$ and $\left[h_{a}, h_{b}\right]$ into four segments: $\left[h_{i-1}, h_{i}\right], i=1,2,3,4$, where $h_{0}=0, h_{1}=$ $h_{a} / 2, h_{2}=h_{a}, h_{3}=h_{a}+\alpha \bar{\tau}$, and $h_{4}=h_{b}, 0<\alpha<1$. For the sake of convenience, we denote $\tau_{i}=h_{i}-h_{i-1}, r_{i}=$ $h_{i}^{2}-h_{i-1}^{2},(i=1,2,3,4)$, and $\bar{\tau}_{0}=h_{a}-0$. For the T-S fuzzy system (4), based on the Lyapunov stability theorem, we will give a stability criterion by using a novel delay decomposition approach as follows.

Theorem 4. (i) In Case I, for given scalars $0<h_{a} \leq h_{b}$, $0<$ $\alpha<1, h_{d}$, the T-S fuzzy system (4) is asymptotically stable if there exist real symmetry matrices $P, Q_{i}>0, R_{i}, Q_{\tau} \geq 0, R_{\tau}$, $\left[\begin{array}{cc}S_{11} & S_{12} \\ * & S_{22}\end{array}\right]>0, Z_{j}=\left[\begin{array}{cc}Z_{j 1} & Z_{j 2} \\ * & Z_{j 3}\end{array}\right](j=1,2)$, and any matrices 
$M_{i}, N_{i},(i=1,2,3,4)$ with appropriate dimensions such that the LMIs in (12) and (13) are feasible. Consider

$$
V_{p}=\left[\begin{array}{ccccc}
P+\sum_{i=1}^{4} \frac{2 \tau_{i}^{3}}{r_{i}} & -\frac{2 \tau_{1}^{2}}{r_{1}} R_{1} & -\frac{2 \tau_{2}^{2}}{r_{2}} R_{2} & -\frac{2 \tau_{3}^{2}}{r_{3}} R_{3} & -\frac{2 \tau_{4}^{2}}{r_{4}} R_{4} \\
* & \frac{1}{\tau_{1}} Q_{1}+\frac{2 \tau_{1}}{r_{1}} R_{1}+\frac{2}{\bar{\tau}_{0}} S_{11} & \frac{2}{\bar{\tau}_{0}} S_{12} & 0 & 0 \\
* & * & \frac{1}{\tau_{2}} Q_{2}+\frac{2 \tau_{2}}{r_{2}} R_{2}+\frac{2}{\bar{\tau}_{0}} S_{22} & 0 & 0 \\
* & * & * & \frac{1}{\tau_{3}} Q_{3}+\frac{2 \tau_{3}}{r_{3}} R_{3} & 0 \\
* & * & * & * & \frac{1}{\tau_{4}} Q_{4}+\frac{2 \tau_{4}}{r_{4}} R_{4}
\end{array}\right]>0,
$$

$$
\Omega(i, k):=\Omega_{0}+\Omega_{1}^{k}+\Omega_{2}^{k}+\Xi_{i}^{k}<0, \quad(i=1,2 ; k=3,4)
$$

$$
\left[\begin{array}{cc}
\tau_{4} R_{4}+\left(1-h_{d}\right) R_{\tau} & {\left[\begin{array}{cc}
M_{4} & N_{4}
\end{array}\right]} \\
Z_{2}
\end{array}\right] \geq 0
$$

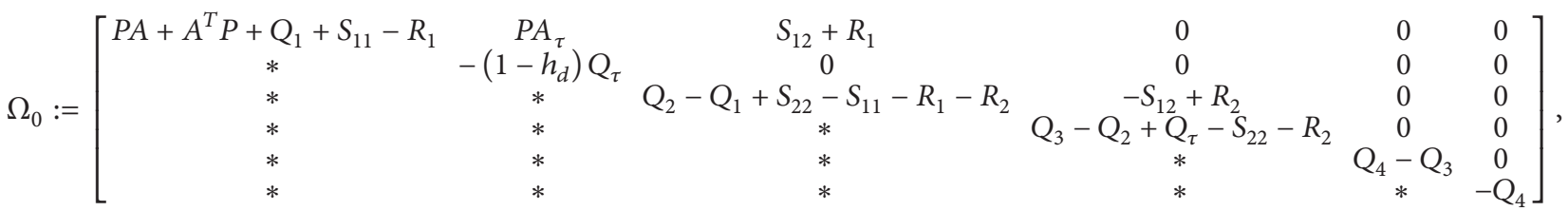

$$
\begin{aligned}
& \Omega_{1}^{3}:=\left[\begin{array}{cccccc}
0 & 0 & 0 & 0 & 0 & 0 \\
* & -N_{1}-N_{1}^{T}+\tau_{3} M_{2}+\tau_{3} M_{2}^{T} & 0 & -M_{1}^{T}+N_{1} & -\tau_{3} M_{2}+\tau_{3} N_{2}^{T} & 0 \\
* & * & 0 & 0 & 0 & 0 \\
* & * & * & M_{1}+M_{1}^{T} & 0 & 0 \\
* & * & * & * & -R_{4}-\tau_{3} N_{2}-\tau_{3} N_{2}^{T} & R_{4} \\
* & * & * & * & * & -R_{4}
\end{array}\right] \\
& \Omega_{1}^{4}:=\left[\begin{array}{cccccc}
0 & 0 & 0 & 0 & 0 & 0 \\
* & -N_{4}-N_{4}^{T}+\tau_{4}\left(M_{3}+M_{3}^{T}\right) & 0 & 0 & -M_{4}^{T}+N_{4} & -\tau_{4} M_{3}+\tau_{4} N_{3}^{T} \\
* & * & 0 & 0 & 0 & 0 \\
* & * & * & M_{1}+M_{1}^{T}+\tau_{3} Z_{11} & -M_{1}+N_{1}^{T}+\tau_{3} Z_{12} & 0 \\
* & * & * & * & -N_{1}-N_{1}^{T}+M_{4}+M_{4}^{T}+\tau_{3} Z_{13} & 0 \\
* & * & * & * & * & -\tau_{4}\left(N_{3}+N_{3}^{T}\right)
\end{array}\right], \\
& \Omega_{2}^{3}:=\Gamma^{T}\left(\sum_{i=1}^{4} \tau_{i}^{2} R_{i}+\tau_{3} R_{\tau}\right) \Gamma, \quad \Omega_{2}^{4}:=\Gamma^{T}\left(\sum_{i=1}^{4} \tau_{i}^{2} R_{i}+\bar{\tau} R_{\tau}\right) \Gamma, \quad \Gamma:=\left[\begin{array}{llllll}
A & A_{\tau} & 0 & 0 & 0 & 0
\end{array}\right], \\
& \Xi_{1}^{k}:=\tau_{k} Z^{k}, \quad Z^{3}:=\left[Z_{i j}^{3}\right]_{6 \times 6}, \quad Z_{i j}^{3} \triangleq\left\{\begin{array}{ll}
Z_{13} & i=2, j=2 \\
Z_{12}^{T} & i=2, j=4 \\
Z_{11} & i=4, j=4 \\
Z_{12} & i=4, j=2 \\
0 & \text { other, }
\end{array} \quad Z^{4}:=\left[Z_{i j}^{4}\right]_{6 \times 6}, \quad Z_{i j}^{4} \triangleq \begin{cases}Z_{23} & i=2, j=2 \\
Z_{22}^{T} & i=2, j=5 \\
Z_{21} & i=5, j=5 \\
Z_{22} & i=5, j=2 \\
0 & \text { other, }\end{cases} \right. \\
& \Xi_{2}^{k}:=\tau_{k}^{2} \Phi^{k} R_{k}^{-1}\left(\Phi^{k}\right)^{T}, \quad \Phi^{3}:=\operatorname{col}\left\{0, M_{2}, 0,0, N_{2}, 0\right\}, \quad \Phi^{4}:=\operatorname{col}\left\{0, M_{3}, 0,0,0, N_{3}\right\} .
\end{aligned}
$$


(ii) In Case II, for given scalars $0<h_{a} \leq h_{b}$ and $0<\alpha<1$, the T-S fuzzy system (4) is asymptotically stable if there exist real symmetry matrices $P, Q_{i}>0, R_{i},\left[\begin{array}{cc}S_{11} & S_{12} \\ * & S_{22}\end{array}\right]>0, Z_{j}=$ $\left[\begin{array}{cc}Z_{j 1} & Z_{j 2} \\ * & Z_{j 3}\end{array}\right](j=1,2)$, and any matrices $M_{i}, N_{i}(i=1,2,3,4)$, with appropriate dimensions such that the LMIs in (12) and (13) with $Q_{\tau}=0, R_{\tau}=0$ are feasible.

Proof. In Case I, choose the following delay-dependent LK functional:

$$
V\left(t, x_{t}\right)=V_{1}\left(t, x_{t}\right)+V_{2}\left(t, x_{t}\right)+V_{3}\left(t, x_{t}\right)+V_{4}\left(t, x_{t}\right),
$$

where $x_{t}$ denotes the function $x(s)$ defined on the interval $\left[t-h_{b}, t\right]$ and

$$
\begin{aligned}
& V_{1}\left(t, x_{t}\right)=x^{T}(t) P x(t), \\
& V_{2}\left(t, x_{t}\right)=\sum_{i=1}^{4} \int_{t-h_{i}}^{t-h_{i-1}} x^{T}(s) Q_{i} x(s) d s \\
& +\int_{t-\tau(t)}^{t-h_{2}} x^{T}(s) Q_{\tau} x(s) d s \\
& V_{3}\left(t, x_{t}\right) \\
& =\int_{t-h_{1}}^{t}\left[x\left(s-\frac{x(s)}{2}\right)\right]^{T}\left[\begin{array}{cc}
S_{11} & S_{12} \\
* & S_{22}
\end{array}\right]\left[x\left(s-\frac{x(s)}{\bar{\tau}_{0}}\right)\right] d s, \\
& V_{4}\left(t, x_{t}\right)=\sum_{i=1}^{4} \tau_{i} \int_{-h_{i}}^{-h_{i-1}} \int_{t+\theta}^{t} \dot{x}^{T}(s) R_{i} \dot{x}(s) d s d \theta \\
& +\int_{-\tau(t)}^{-h_{2}} \int_{t+\theta}^{t} \dot{x}^{T}(s) R_{\tau} \dot{x}(s) d s d \theta .
\end{aligned}
$$

with $P, Q_{i}>0(i=1,2,3,4), Q_{\tau} \geq 0,\left[\begin{array}{cc}S_{11} & S_{12} \\ * & S_{22}\end{array}\right]>0, R_{i}(i=$ $1,2,3,4), R_{\tau}$ being real symmetry matrices.
First, we show that the condition $V\left(t, x_{t}\right) \geq \varepsilon_{1}\|x(t)\|,\left(\varepsilon_{1}>\right.$ $0)$ is satisfied. Assume that $Q_{i}>0$ and $R_{i}>0(i=1,2,3,4)$; then, suitably applying Lemma 1 yields

$$
\begin{aligned}
V\left(t, x_{t}\right) \geq & x^{T}(t) P x(t)+\int_{t-\tau(t)}^{t-h_{2}} x^{T}(s) Q_{\tau} x(s) d s \\
& +\int_{-\tau(t)}^{-h_{2}} \int_{t+\theta}^{t} \dot{x}^{T}(s) R_{\tau} \dot{x}(s) d s d \theta \\
& +\sum_{i=1}^{4} \frac{1}{\tau_{i}} \int_{t-h_{i}}^{t-h_{i-1}} x^{T}(s) d s Q_{i} \int_{t-h_{i}}^{t-h_{i-1}} x(s) d s \\
& +\int_{t-h_{1}}^{t}\left[x\left(s-\frac{\bar{\tau}_{0}}{2}\right)\right]^{T} d s\left[\begin{array}{ll}
S_{11} & S_{12} \\
* & S_{22}
\end{array}\right] \\
& \times \int_{t-h_{1}}^{t}\left[x\left(s-\frac{\bar{\tau}_{0}}{2}\right)\right] d s \\
& +\sum_{i=1}^{4} \frac{2 \tau_{i}}{r_{i}} \int_{-h_{i}}^{-h_{i-1}} \int_{t+\theta}^{t} \dot{x}^{T}(s) d s d \theta \\
& \left.\times R_{i} \int_{-h_{i}}^{-h_{i-1}}\right]_{t+\theta}^{t} \dot{x}(s) d s d \theta .
\end{aligned}
$$

We denote $\zeta(t):=\operatorname{col}\left\{x(t) \int_{t-h_{1}}^{t} x(s) d s \int_{t-h_{2}}^{t-h_{1}} x(s) d s\right.$ $\left.\int_{t-h_{3}}^{t-h_{2}} x(s) d s \int_{t-h_{4}}^{t-h_{3}} x(s) d s\right\}$; after the simple arrangement, we have

$$
\begin{aligned}
V\left(t, x_{t}\right) \geq & \zeta^{T}(t) V_{p} \zeta(t)+\int_{t-\tau(t)}^{t-h_{2}} x^{T}(s) Q_{\tau} x(s) d s \\
& +\int_{-\tau(t)}^{-h_{2}} \int_{t+\theta}^{t} \dot{x}^{T}(s) R_{\tau} \dot{x}(s) d s d \theta,
\end{aligned}
$$

where
Therefore, if the right side of (19) is positive, it yields a sufficient condition to guarantee that $V\left(t, x_{t}\right) \geq \varepsilon_{1}\|x(t)\|,\left(\varepsilon_{1}>0\right)$. Moreover, the LMI in (12) implies that $R_{i}>0(i=1,2,3,4)$ and $R_{\tau}>0$. Consequently, if the LMIs in (7) are satisfied, $Q_{i}>0(i=1,2,3,4)$, and $Q_{\tau} \geq 0$, then $V\left(t, x_{t}\right) \geq$ $\varepsilon_{1}\|x(t)\|,\left(\varepsilon_{1}>0\right)$. 
Secondly, we show that the condition $\dot{V}\left(t, x_{t}\right) \leq$ $-\varepsilon_{2}\|x(t)\|\left(\varepsilon_{2}>0\right)$ is also guaranteed if the LMIs in (13) and $Q_{\tau} \geq 0, Q_{i}>0(i=1,2,3,4),\left[\begin{array}{cc}S_{11} & S_{12} \\ * & S_{22}\end{array}\right]>0$ hold. Taking the derivative of (16) with respect to $t$ along the trajectory of system (4), we have

$$
\begin{aligned}
& \dot{V}_{1}\left(t, x_{t}\right)=\dot{x}^{T}(t) P x(t)+x^{T}(t) P \dot{x}(t) \\
& =\left[A x(t)+A_{\tau} x(t-\tau(t))\right]^{T} P x(t) \\
& +x^{T}(t) P\left[A x(t)+A_{\tau} x(t-\tau(t))\right], \\
& \dot{V}_{2}\left(t, x_{t}\right)=\sum_{i=1}^{4}\left[x^{T}\left(t-h_{i-1}\right) Q_{i} x\left(t-h_{i-1}\right)\right. \\
& \left.-x^{T}\left(t-h_{i}\right) Q_{i} x\left(t-h_{i}\right)\right] \\
& +x^{T}\left(t-h_{2}\right) Q_{\tau} x\left(t-h_{2}\right)-(1-\dot{\tau}(t)) x^{T} \\
& \times(t-\tau(t)) Q_{\tau} x(t-\tau(t)) \\
& \leq \sum_{i=1}^{4}\left[x^{T}\left(t-h_{i-1}\right) Q_{i} x\left(t-h_{i-1}\right)\right. \\
& \left.-x^{T}\left(t-h_{i}\right) Q_{i} x\left(t-h_{i}\right)\right] \\
& +x^{T}\left(t-h_{2}\right) Q_{\tau} x\left(t-h_{2}\right) \\
& -\left(1-h_{d}\right) x^{T}(t-\tau(t)) Q_{\tau} x(t-\tau(t)) \text {, } \\
& \dot{V}_{3}\left(t, x_{t}\right)=\left[\begin{array}{c}
x(t) \\
x\left(t-h_{1}\right)
\end{array}\right]^{T}\left[\begin{array}{ll}
S_{11} & S_{12} \\
* & S_{22}
\end{array}\right]\left[\begin{array}{c}
x(t) \\
x\left(t-h_{1}\right)
\end{array}\right] \\
& -\left[\begin{array}{c}
x\left(t-h_{1}\right) \\
x\left(t-h_{2}\right)
\end{array}\right]^{T}\left[\begin{array}{cc}
S_{11} & S_{12} \\
* & S_{22}
\end{array}\right]\left[\begin{array}{c}
x\left(t-h_{1}\right) \\
x\left(t-h_{2}\right)
\end{array}\right], \\
& \dot{V}_{4}\left(t, x_{t}\right)=\sum_{i=1}^{4} \tau_{i}^{2} \dot{x}^{T}(t) R_{i} \dot{x}(t) \\
& +\left(\tau(t)-h_{2}\right) \dot{x}^{T}(t) R_{\tau} \dot{x}(t) \\
& -\sum_{i=1}^{4} \tau_{i} \int_{t-h_{i}}^{t-h_{i-1}} \dot{x}^{T}(s) R_{i} \dot{x}(s) d s \\
& -(1-\dot{\tau}(t)) \int_{t-\tau(t)}^{t-h_{2}} \dot{x}^{T}(s) R_{\tau} \dot{x}(s) d s .
\end{aligned}
$$

For any $t \geq 0$, it is a fact that $h_{a} \leq \tau(t) \leq h_{a}+\alpha \tau$ or $h_{a}+\alpha \tau \leq \tau(t) \leq h_{b},(0<\alpha<1)$. In the case of $h_{a} \leq \tau(t) \leq h_{a}+$ $\alpha \tau$, that is, $\tau(t) \in\left[h_{2}, h_{3}\right], k=3$, suitably using the integral inequalities in Lemma 1 , the following inequalities are true:

$$
\begin{aligned}
& \left(\tau(t)-h_{2}\right) \dot{x}^{T}(t) R_{\tau} \dot{x}(t) \\
& \quad \leq \alpha \bar{\tau} \dot{x}^{T}(t) R_{\tau} \dot{x}(t)=\tau_{3} \dot{x}^{T}(t) R_{\tau} \dot{x}(t),
\end{aligned}
$$

$$
\begin{aligned}
& -\tau_{i} \int_{t-h_{i}}^{t-h_{i-1}} \dot{x}^{T}(s) R_{i} \dot{x}(s) d s \\
& \leq\left[\begin{array}{c}
x\left(t-h_{i-1}\right) \\
x\left(t-h_{i}\right)
\end{array}\right]^{T}\left[\begin{array}{cc}
-R_{i} & R_{i} \\
* & -R_{i}
\end{array}\right]\left[\begin{array}{c}
x\left(t-h_{i-1}\right) \\
x\left(t-h_{i}\right)
\end{array}\right], \\
& (i=1,2,4) \text {, } \\
& -\tau_{3} \int_{t-h_{3}}^{t-h_{2}} \dot{x}^{T}(s) R_{3} \dot{x}(s) d s \\
& -(1-\dot{\tau}(t)) \int_{t-\tau(t)}^{t-h_{2}} \dot{x}^{T}(s) R_{\tau} \dot{x}(s) d s \\
& \leq-\tau_{3} \int_{t-h_{3}}^{t-h_{2}} \dot{x}^{T}(s) R_{3} \dot{x}(s) d s \\
& -\left(1-h_{d}\right) \int_{t-\tau(t)}^{t-h_{2}} \dot{x}^{T}(s) R_{\tau} \dot{x}(s) d s \\
& =-\int_{t-\tau(t)}^{t-h_{2}} \dot{x}^{T}(s)\left(\tau_{3} R_{3}+\left(1-h_{d}\right) R_{\tau}\right) \dot{x}(s) d s \\
& -\tau_{3} \int_{t-h_{3}}^{t-\tau(t)} \dot{x}^{T}(s) R_{3} \dot{x}(s) d s \\
& \leq\left[\begin{array}{c}
x\left(t-h_{2}\right) \\
x(t-\tau(t))
\end{array}\right]^{T}\left\{\left[\begin{array}{cc}
M_{1}+M_{1}^{T} & -M_{1}+N_{1}^{T} \\
* & -N_{1}-N_{1}^{T}
\end{array}\right]+\rho \cdot \alpha \bar{\tau} \cdot Z_{1}\right\} \\
& \times\left[\begin{array}{c}
x\left(t-h_{2}\right) \\
x(t-\tau(t))
\end{array}\right] \\
& +\left[\begin{array}{c}
x(t-\tau(t)) \\
x\left(t-h_{3}\right)
\end{array}\right]^{T}\left\{\tau_{3}\left[\begin{array}{cc}
M_{2}+M_{2}^{T} & -M_{2}+N_{2}^{T} \\
* & -N_{2}-N_{2}^{T}
\end{array}\right]\right. \\
& \left.+(1-\rho) \cdot \alpha \bar{\tau} \cdot\left[\begin{array}{c}
M_{2} \\
N_{2}
\end{array}\right] R_{3}^{-1}\left[\begin{array}{c}
M_{2} \\
N_{2}
\end{array}\right]^{T}\right\} \\
& \times\left[\begin{array}{c}
x(t-\tau(t)) \\
x\left(t-h_{3}\right)
\end{array}\right]
\end{aligned}
$$

with $\left[\begin{array}{cc}\tau_{3} R_{3}+\left(1-h_{d}\right) R_{\tau} & {\left[\begin{array}{cc}M_{1} & N_{1}\end{array}\right]} \\ Z_{1}\end{array}\right] \geq 0$, and $\rho=((\tau(t)-$ $\left.\left.h_{2}\right) / \alpha \bar{\tau}\right), 0 \leq \rho \leq 1$.

It follows from (21)-(27) that

$$
\begin{aligned}
& \dot{V}\left(t, x_{t}\right) \\
& \leq \rho \xi^{T}(t)\left[\Omega_{0}+\Omega_{1}^{3}+\Gamma^{T}\left(\sum_{i=1}^{4} \tau_{i}^{2} R_{i}+\alpha \bar{\tau} R_{\tau}\right) \Gamma+\alpha \bar{\tau} Z^{3}\right] \\
& \times \xi(t)+(1-\rho) \xi^{T}(t) \\
& \quad \times\left[\Omega_{0}+\Omega_{1}^{3}+\Gamma^{T}\left(\sum_{i=1}^{4} \tau_{i}^{2} R_{i}+\alpha \bar{\tau} R_{\tau}\right) \Gamma\right. \\
& \left.+(\alpha \bar{\tau})^{2} \Phi^{3} R_{3}^{-1}\left(\Phi^{3}\right)^{T}\right] \xi(t)
\end{aligned}
$$


with $\left[\begin{array}{cc}\tau_{3} R_{3}+\left(1-h_{d}\right) R_{\tau} & {\left[\begin{array}{cc}M_{1} & N_{1}\end{array}\right]} \\ Z_{1}\end{array}\right] \geq 0$ where $\Omega_{0}, \Omega_{1}^{3}, \Gamma, Z^{3}$, and $\Phi^{3}$ are defined in (15), and $\xi(t)$ := $\operatorname{col}\left\{x(t) \quad x(t-\tau(t)) \quad x\left(t-h_{1}\right) \quad x\left(t-h_{2}\right) \quad x\left(t-h_{3}\right)\right.$ $\left.x\left(t-h_{4}\right)\right\}$.

Since $0 \leq \rho \leq 1$, applying the convex combination method, we conclude that if the following LMIs

$$
\begin{aligned}
\Omega_{0}+ & \Omega_{1}^{3}+\Gamma^{T}\left(\sum_{i=1}^{4} \tau_{i}^{2} R_{i}+\alpha \bar{\tau} R_{\tau}\right) \Gamma+\alpha \bar{\tau} Z^{3}<0, \\
\Omega_{0} & +\Omega_{1}^{3}+\Gamma^{T}\left(\sum_{i=1}^{4} \tau_{i}^{2} R_{i}+\alpha \bar{\tau} R_{\tau}\right) \Gamma \\
& +(\alpha \bar{\tau})^{2} \Phi^{3} R_{3}^{-1}\left(\Phi^{3}\right)^{T}<0
\end{aligned}
$$

are simultaneously feasible, then $\dot{V}\left(t, x_{t}\right)<-\varepsilon_{2}\|x(t)\|$ is true for a sufficiently small $\varepsilon_{2}>0$.

Meanwhile, if $h_{a}+\alpha \tau \leq \tau(t) \leq h_{b}$, that is, $\tau(t) \in\left[h_{3}, h_{4}\right]$, $k=4$, similar to the above deduction process, we also can obtain similar stability conditions.

In Case II, a Lyapunov functional can be chosen as (16) with $Q_{\tau}=0, R_{\tau}=0$. Similar to the above analysis, one can get that $\dot{V}\left(t, x_{t}\right)<-\varepsilon_{2}\|x(t)\|\left(\varepsilon_{2}>0\right)$.

Thus, this completes the proof.

Remark 5. It may be noted that, in the above, no approximation of the delay term is involved excepting exploiting a convex combination of the uncertain terms involved. In fact, Lemma 1 plays a key effect on the present results, which is different from the common Jensen's inequality, although their similarity can be established following the equivalency results in [31]. However, if $h=\tau_{2}-\tau_{1}:=h(t)$ is uncertain and is required to be approximated with its lower or upper bound then use of (9) or (10) would be beneficial since the free variables $M, N$ are introduced. Such a feature leads to less conservative results compared to the existing ones as is shown in the next section using numerical examples.

When the lower bound of the delay is 0 , that is, $h_{a}=0$, and the interval team $\left[0, h_{a}\right]$ is missing, we may set $Q_{1}=0$, $Q_{2}=0, S_{i j}=0, R_{1}=0$, and $R_{2}=0$ in (16), according to Theorem 4, and then obtain Corollary 6.

Corollary 6. (i) In Case 1, for given scalars $0=h_{a} \leq h_{b}, 0<$ $\alpha<1, h_{d}$, the T-S fuzzy systems (4) are asymptotically stable if there exist real symmetric matrices $P, Q_{i}>0, R_{i}(i=3,4)$, $Q_{\tau} \geq 0, R_{\tau}, Z_{i}=\left[\begin{array}{cc}Z_{i 1} & Z_{i 2} \\ * & Z_{i 3}\end{array}\right](i=1,2)$, and any matrices $M_{i}, N_{i}(i=1,2,3,4)$ with appropriate dimensions such that the LMIs in (30) are feasible as follows:

$$
\begin{aligned}
& \widehat{V}_{p}=\left[\begin{array}{ccc}
P+\sum_{i=3}^{4} \frac{2 \tau_{i}^{3}}{r_{i}} R_{i} & -\frac{2 \tau_{3}^{2}}{r_{3}} R_{3} & -\frac{2 \tau_{4}^{2}}{r_{4}} R_{4} \\
* & \frac{1}{\tau_{3}} Q_{3}+\frac{2 \tau_{3}}{r_{3}} R_{3} & 0 \\
* & * & \frac{1}{\tau_{4}} Q_{4}+\frac{2 \tau_{4}}{r_{4}} R_{4}
\end{array}\right] \\
& >0, \\
& \widehat{\Omega}(i, k):=\widehat{\Omega}_{0}+\widehat{\Omega}_{1}^{k}+\widehat{\Omega}_{2}^{k}+\widehat{\Xi}_{i}^{k}<0, \quad(i=1,2 ; k=3,4)
\end{aligned}
$$

with

$$
\begin{aligned}
& {\left[\begin{array}{cc}
\tau_{3} R_{3}+\left(1-h_{d}\right) R_{\tau} & {\left[\begin{array}{cc}
M_{1} & N_{1}
\end{array}\right]} \\
* & Z_{1}
\end{array}\right] \geq 0,} \\
& {\left[\begin{array}{cc}
\tau_{4} R_{4}+\left(1-h_{d}\right) R_{\tau} & {\left[\begin{array}{cc}
M_{4} & N_{4}
\end{array}\right]} \\
Z_{2}
\end{array}\right] \geq 0,}
\end{aligned}
$$

where

$$
\begin{aligned}
& \widehat{\Omega}_{0}:=\left[\begin{array}{cccc}
P A+A^{T} P+Q_{\tau}+Q_{3} & P A_{\tau} & 0 & 0 \\
* & \left(1-h_{d}\right) Q_{\tau} & 0 & 0 \\
* & * & Q_{4}-Q_{3} & 0 \\
* & * & * & -Q_{4}
\end{array}\right] \\
& \widehat{\Omega}_{1}^{3}:=\left[\begin{array}{cccc}
M_{1}+M_{1}^{T} & -M_{1}+N_{1}^{T} & 0 & 0 \\
* & -N_{1}-N_{1}^{T}+\tau_{3} M_{2}+\tau_{3} M_{2}^{T} & -\tau_{3} M_{2}+\tau_{3} N_{2}^{T} & 0 \\
* & * & R_{4}-\tau_{3} N_{2}-\tau_{3} N_{2}^{T} & R_{4} \\
* & * & * & -R_{4}
\end{array}\right] \\
& \widehat{\Omega}_{1}^{4}:=\left[\begin{array}{cccc}
M_{1}+M_{1}^{T}+\tau_{3} Z_{11} & 0 & -M_{1}+N_{1}^{T}+\tau_{3} Z_{12} & 0 \\
* & -N_{4}-N_{4}^{T}+\tau_{4}\left(M_{3}+M_{3}^{T}\right) & -M_{4}^{T}+N_{4} & -\tau_{4} M_{3}+\tau_{4} N_{3}^{T} \\
* & * & -N_{1}-N_{1}^{T}+M_{4}+M_{4}^{T}+\tau_{3} Z_{13} & 0 \\
* & * & * & -\tau_{4}\left(N_{3}+N_{3}^{T}\right)
\end{array}\right] \text {, } \\
& \widehat{\Omega}_{2}^{3}:=\widehat{\Gamma}^{T}\left(\sum_{i=3}^{4} \tau_{i}^{2} R_{i}+\tau_{3} R_{\tau}\right) \widehat{\Gamma}, \quad \widehat{\Omega}_{2}^{4}:=\widehat{\Gamma}^{T}\left(\sum_{i=3}^{4} \tau_{i}^{2} R_{i}+\bar{\tau} R_{\tau}\right) \widehat{\Gamma}, \quad \widehat{\Gamma}:=\left[\begin{array}{llll}
A & A_{\tau} & 0 & 0
\end{array}\right],
\end{aligned}
$$




$$
\begin{gathered}
\widehat{\Xi}_{1}^{k}:=\tau_{k} \widehat{Z}^{k}, \quad(k=3,4), \quad \widehat{Z}^{3}:=\left[\widehat{Z}_{i j}^{3}\right]_{4 \times 4} \quad \widehat{Z}_{i j}^{3} \triangleq \begin{cases}Z_{11} & i=1, j=1 \\
Z_{12} & i=1, j=2 \\
Z_{12}^{T} & i=2, j=1 \\
Z_{13} & i=2, j=2 \\
0 & \text { other, }\end{cases} \\
\widehat{Z}^{4}:=\left[\widehat{Z}_{i j}^{4}\right]_{4 \times 4}, \quad \widehat{Z}_{i j}^{4} \triangleq \begin{cases}Z_{23} & i=2, j=2 \\
Z_{22}^{T} & i=2, j=3 \\
Z_{21} & i=3, j=3 \\
Z_{22} & i=3, j=2 \\
0 \quad \text { other, }\end{cases} \\
\widehat{\Xi}_{2}^{k}:=\tau_{k}^{2} \widehat{\Phi}^{k} R_{k}^{-1}\left(\widehat{\Phi}^{k}\right)^{T}, \quad(k=3,4), \quad \widehat{\Phi}^{3}:=\operatorname{col}\left\{0, M_{2}, N_{2}, 0\right\}, \\
\widehat{\Phi}^{4}:=\operatorname{col}\left\{0, M_{3}, 0, N_{3}\right\} .
\end{gathered}
$$

(ii) In Case 2, if the LMIs in (30) with $Q_{\tau}=0$ and $R_{\tau}=0$ are feasible, the aforementioned system is asymptotically stable for fast-varying delay.

Notice that Theorem 4 and Corollary 6 still have some free-weighting matrices. In the following we present the second stability criterion in which no free-weighting matrix is involved.

Similar to the line of derivation process of Theorem 4, for (27), we have

$$
\begin{aligned}
& -\tau_{3} \int_{t-h_{3}}^{t-h_{2}} \dot{x}^{T}(s) R_{3} \dot{x}(s) d s \\
& -(1-\dot{\tau}(t)) \int_{t-\tau(t)}^{t-h_{2}} \dot{x}^{T}(s) R_{\tau} \dot{x}(s) d s \\
& \leq-\tau_{3} \int_{t-h_{3}}^{t-h_{2}} \dot{x}^{T}(s) R_{3} \dot{x}(s) d s \\
& -\left(1-h_{d}\right) \int_{t-\tau(t)}^{t-h_{2}} \dot{x}^{T}(s) R_{\tau} \dot{x}(s) d s \\
& =-\int_{t-\tau(t)}^{t-h_{2}} \dot{x}^{T}(s)\left[\tau_{3} R_{3}+\left(1-h_{d}\right) R_{\tau}\right] \dot{x}(s) d s \\
& -\tau_{3} \int_{t-h_{3}}^{t-\tau(t)} \dot{x}^{T}(s) R_{3} \dot{x}(s) d s \\
& \leq-\frac{h_{3}-h_{2}}{\tau(t)-h_{2}}\left(x^{T}\left(t-h_{2}\right)-x^{T}(t-\tau(t))\right) \\
& \times\left(R_{3}+\frac{\left(1-h_{d}\right)}{\tau_{3}} R_{\tau}\right)\left(x\left(t-h_{2}\right)-x(t-\tau(t))\right) \\
& -\frac{h_{3}-h_{2}}{h_{3}-\tau(t)}\left(x^{T}(t-\tau(t))-x^{T}\left(t-h_{3}\right)\right) \\
& \times\left(R_{3}\right)\left(x(t-\tau(t))-x\left(t-h_{3}\right)\right) .
\end{aligned}
$$

Based on Lemma 3, if we denote

$$
\begin{aligned}
M \triangleq & \left(x^{T}\left(t-h_{2}\right)-x^{T}(t-\tau(t))\right) \\
& \times\left(R_{3}+\frac{\left(1-h_{d}\right)}{\tau_{3}} R_{\tau}\right)\left(x\left(t-h_{2}\right)-x(t-\tau(t))\right), \\
N \triangleq & \left(x^{T}(t-\tau(t))-x^{T}\left(t-h_{3}\right)\right)\left(R_{3}\right) \\
& \times\left(x(t-\tau(t))-x\left(t-h_{3}\right)\right),
\end{aligned}
$$

then we have $-\tau_{3} \int_{t-h_{3}}^{t-h_{2}} \dot{x}^{T}(s) R_{3} \dot{x}(s) d s-(1-\dot{\tau}(t))$ $\int_{t-\tau(t)}^{t-h_{2}} \dot{x}^{T}(s) R_{\tau} \dot{x}(s) d s \leq \max \{-(M+3 N),-(N+3 M)\}$. After rearrangement of (21)-(26) and (33), we can deduce a similar formula as (28)-(29) and then obtain the following result for the T-S fuzzy system (4).

Theorem 7. (i) In Case 1, for given scalars $0<h_{a} \leq h_{b}, 0<$ $\alpha<1, h_{d}$, the T-S fuzzy systems (4) are asymptotically stable if there exist real symmetric matrices $P, Q_{i}>0, R_{i}(i=1,2,3,4)$, $Q_{\tau} \geq 0, R_{\tau},\left[\begin{array}{cc}S_{11} & S_{12} \\ * & S_{22}\end{array}\right]>0$ such that the LMIs in (12) and (35) are feasible. Consider

$$
\widetilde{\Omega}(i, k):=\Omega_{0}+\Omega_{2}^{k}+\widetilde{\Xi}_{i}^{k}<0 \quad(i=1,2 ; k=3,4)
$$

with $\tau_{3} R_{3}+\left(1-h_{d}\right) R_{\tau}>0$ and $\tau_{4} R_{4}+\left(1-h_{d}\right) R_{\tau}>0$, where the teams are defined in (15) except that $\widetilde{\Xi}_{i}^{k}$ are described as follows: 


$$
\begin{aligned}
& \widetilde{\Xi}_{1}^{3}:=\left[\begin{array}{cccccc}
0 & 0 & 0 & 0 & 0 & 0 \\
* & -4 R_{3}-\frac{1-h_{d}}{\tau_{3}} R_{\tau} & 0 & R_{3}+\frac{1-h_{d}}{\tau_{3}} R_{\tau} & 3 R_{3} & 0 \\
* & * & 0 & 0 & 0 & 0 \\
* & * & * & -R_{3}-\frac{1-h_{d}}{\tau_{3}} R_{\tau} & 0 & 0 \\
* & * & * & * & -R_{4}-3 R_{3} & R_{4} \\
* & * & * & * & * & -R_{4}
\end{array}\right] \\
& \widetilde{\Xi}_{2}^{3}:=\left[\begin{array}{cccccc}
0 & 0 & 0 & 0 & 0 & 0 \\
* & -4 R_{3}-\frac{3\left(1-h_{d}\right)}{\tau_{3}} R_{\tau} & 0 & 3 R_{3}+\frac{3\left(1-h_{d}\right)}{\tau_{3}} R_{\tau} & R_{3} & 0 \\
* & * & 0 & 0 & 0 & 0 \\
* & * & * & -3 R_{3}-\frac{3\left(1-h_{d}\right)}{\tau_{3}} R_{\tau} & 0 & 0 \\
* & * & * & * & -R_{4}-R_{3} & R_{4} \\
* & * & * & * & * & -R_{4}
\end{array}\right], \\
& \widetilde{\Xi}_{1}^{4}:=\left[\begin{array}{cccccc}
0 & 0 & 0 & 0 & 0 & 0 \\
* & -4 R_{4}-\frac{1-h_{d}}{\tau_{4}} R_{\tau} & 0 & 0 & R_{4}+\frac{1-h_{d}}{\tau_{4}} R_{\tau} & 3 R_{4} \\
* & * & 0 & 0 & 0 & 0 \\
* & * & * & -R_{3}-\frac{1-h_{d}}{\tau_{3}} R_{\tau} & R_{3}+\frac{1-h_{d}}{\tau_{3}} R_{\tau} & 0 \\
* & * & * & * & -R_{3}-\frac{1-h_{d}}{\tau_{3}} R_{\tau}-R_{4}-\frac{1-h_{d}}{\tau_{4}} R_{\tau} & 0 \\
* & * & * & * & * & -3 R_{4}
\end{array}\right], \\
& \widetilde{\Xi}_{2}^{4}:=\left[\begin{array}{cccccc}
0 & 0 & 0 & 0 & 0 & 0 \\
* & -4 R_{4}-\frac{3\left(1-h_{d}\right)}{\tau_{4}} R_{\tau} & 0 & 0 & 3 R_{4}+\frac{3\left(1-h_{d}\right)}{\tau_{4}} R_{\tau} & R_{4} \\
* & * & 0 & 0 & 0 & 0 \\
* & * & * & -R_{3}-\frac{1-h_{d}}{\tau_{3}} R_{\tau} & R_{3}+\frac{1-h_{d}}{\tau_{3}} R_{\tau} & 0 \\
* & * & * & * & -R_{3}-\frac{1-h_{d}}{\tau_{3}} R_{\tau}-3 R_{4}-\frac{3\left(1-h_{d}\right)}{\tau_{4}} R_{\tau} & 0 \\
* & * & * & * & * & -R_{4}
\end{array}\right] .
\end{aligned}
$$

(ii) In Case 2, if the LMIs in (12) and (35) with $Q_{\tau}=$ 0 and $R_{\tau}=0$ are feasible, the aforementioned system is asymptotically stable for fast-varying delay.

We have proposed two techniques to investigate the stability of T-S fuzzy system (4) and obtain two stability results, Theorems 4 and 7, both less conservative with smaller numerical complexity than the existing ones. The two techniques are different mainly in the way of estimating the cross terms. The first estimation has no conservatism but involves seven free-weighting matrices, which are introduced to make use of the constraints $M_{i}, N_{i}(i=1,2,3,4)$ and
$Z_{1}, Z_{2}$. By contrast, the second estimation involves no freeweighting matrices though a little more conservative. This ends in that Theorem 7 has smaller numerical complexity than Theorem 4.

Remark 8. By constructing new augmented LKF (16) and using useful inequalities in Lemmas 1 and 3, Theorems 4 and 7 are obtained with and without using any free matrices, respectively, which are dependent on $\tau_{i}, r_{i}(i=1,2,3,4)$ with relation to a tuning parameter. Moreover, the proposed results can be extended to robust $H_{-} / H_{\infty}$ control and fault detection/fault tolerant tracking control for T-S systems with 
unknown inputs or input constraints and with unmeasurable premise variables as [19-22], robust observer and output feedback control of the above systems [15, 23], NCSs, and multidelays systems, and the corresponding results will appear in the near future.

\section{Numerical Examples}

This section gives three examples to demonstrate the effectiveness and reduced conservatism of the proposed approach. For comparisons, we study T-S fuzzy system (4) with fuzzy rules investigated in recent publications $[6-10,12-14,26]$.

Example 1 (Example 1 of [7]). Consider a two-rule system:

$$
\dot{x}(t)=\sum_{i=1}^{2} h_{i}\left[A_{i} x(t)+A_{\tau i} x(t-\tau(t))\right],
$$

where $h_{1}=1 /\left(1+\exp \left(-2 x_{1}(t)\right)\right), h_{2}=1-h_{1}$, and

$$
\begin{array}{ll}
A_{1}=\left[\begin{array}{cc}
-2 & 0 \\
0 & -0.9
\end{array}\right], & A_{\tau 1}=\left[\begin{array}{cc}
-1 & 0 \\
-1 & -1
\end{array}\right], \\
A_{2}=\left[\begin{array}{cc}
-1.5 & 1 \\
0 & -0.75
\end{array}\right], & A_{\tau 2}=\left[\begin{array}{cc}
-1 & 0 \\
1 & -0.85
\end{array}\right] .
\end{array}
$$

For this T-S fuzzy system, it is easy to notice, from Table 1, that the proposed result asserts the system's stability with the larger MADB to the time-varying delay with unknown time-derivative. If the additional information $h_{d}=0.3$ is given, larger upper bounds of the delay can be computed by Theorems 4 and 7 for various tuning delay-fractional parameters $\alpha$, respectively, which are shown in Table 2. Moreover, when $h_{d}=0.5$, the maximum allowable delay bound (MADB) $h_{b}$ obtained from the criterion [28] are 1.3451 and 1.4016 with $h_{a}=0.1$ and $h_{a}=0.3$, while Theorem 4 $(\alpha=0.65)$ in this paper can compute the MADB as 1.5503 and 1.4874 with $h_{a}=0.1$ and $h_{a}=0.3$, respectively.

It is worth mentioning that Theorems 4 and 7 can provide larger upper bound than the existing ones. At the same time, Theorem 7 has some larger conservative than Theorem 4 but has less numerical complexity than some existing ones since Theorem 7 does not involve any free matrices. Next, we will give another example to illustrate the merit of our method, which is reduced conservative than the existing results.

Example 2 (Example 1 of [26]). Consider a two-rule system:

$$
\dot{x}(t)=\sum_{i=1}^{2} h_{i}\left[A_{i} x(t)+A_{\tau i} x(t-\tau(t))\right],
$$

where $h_{1}=1 /\left(1+\exp \left(-2 x_{1}(t)\right)\right), h_{2}=1-h_{1}$, and

$$
\begin{array}{ll}
A_{1}=\left[\begin{array}{cc}
-2.1 & 0.1 \\
-0.2 & -0.9
\end{array}\right], & A_{2}=\left[\begin{array}{cc}
-1.9 & 0 \\
-0.2 & -1.1
\end{array}\right], \\
A_{\tau 1}=\left[\begin{array}{cc}
-1.1 & 0.1 \\
-0.8 & -0.9
\end{array}\right], & A_{\tau 2}=\left[\begin{array}{cc}
-0.9 & 0 \\
-1.1 & -1.2
\end{array}\right] .
\end{array}
$$

TABLE 1: Comparison of MADB $h_{b}$ for various $h_{a}$ with unknown delay derivative $h_{d}$.

\begin{tabular}{lcccc}
\hline Method $\backslash h_{a}$ & 0.2 & 0.4 & 0.6 & 0.8 \\
\hline Lien et al. [6] & 0.7945 & 0.8487 & 0.9316 & 1.0480 \\
Peng et al. [8] & 0.9119 & 0.9793 & 1.0639 & 1.1662 \\
Tian et al. [7] & 1.1410 & 1.1500 & 1.1720 & 1.2090 \\
Souza et al. [10] & 1.1639 & 1.1734 & 1.1994 & 1.2532 \\
Theorem 7 $(\alpha=0.55)$ & 1.1640 & 1.1844 & 1.2100 & 1.2567 \\
Theorem 4 $(\alpha=0.6)$ & 1.4618 & 1.4202 & 1.4005 & 1.4095 \\
\hline
\end{tabular}

TABLE 2: Comparison of MADB $h_{b}$ for various $h_{a}$ with $h_{d}=0.3$.

\begin{tabular}{lcccc}
\hline Method $\backslash h_{a}$ & 0.2 & 0.4 & 0.6 & 0.8 \\
\hline Theorem 7 $(\alpha=0.5)$ & 1.2872 & 1.3018 & 1.3155 & 1.3230 \\
Theorem 7 $(\alpha=0.55)$ & 1.2842 & 1.3018 & 1.3165 & 1.3234 \\
Theorem 4 $(\alpha=0.6)$ & 1.5934 & 1.5535 & 1.4999 & 1.4510 \\
Theorem 4 $(\alpha=0.65)$ & 1.6013 & 1.5485 & 1.4882 & 1.4381 \\
\hline
\end{tabular}

TABLE 3: MADB on $h_{b}$ for various delay derivatives $h_{d}$ and $h_{a}$.

\begin{tabular}{lcccc}
\hline$h_{d} \backslash h_{a}$ & 0 & 1 & 2 & 3 \\
\hline 0.1 & 3.288 & 3.874 & 3.774 & 3.480 \\
0.2 & 2.819 & 3.229 & 3.013 & 3.252 \\
0.3 & 2.494 & 2.754 & 2.566 & 3.252 \\
1.1 & 1.779 & 2.031 & 2.563 & 3.252 \\
$h_{d}$ unknown & 1.779 & 2.031 & 2.563 & 3.252 \\
\hline
\end{tabular}

Choosing $h_{d}$ and $h_{a}$ in Table 3 and applying Theorem 4 ( $\alpha=0.7)$ and Corollary $6(\alpha=0.8)$, the larger MADB on $h_{b}$ of the delay, which guarantees the asymptotic stability of the fuzzy system, is listed in Table 3 . Moreover, when $h_{d}$ are unknown, that is, fast-varying delay case, by setting $Q_{\tau}=0$ and $R_{\tau}=0$, the obtained $h_{b}$ are computed as shown in the last column of Table 3. It is worthwhile to point out that, when $h_{d} \geq 1$, the $Q_{\tau}, R_{\tau}$ in LK functional authentically will no longer be helpful to improve the stability criterion.

To compare with the existing results in $[14,25,26]$, we assume that $h_{a}=0$. Using Corollary 6 yields larger MADB on $h_{b}$ that guarantees the stability of fuzzy system for various $h_{d}$, which are listed in Table 4 . From Table 4, it can be seen that when the delay is time-invariant, that is, $h_{d}=0$, the obtained results in Corollary 6 are better than those in $[14,25,26]$. Furthermore, when the delay is time-varying, the authors in [25] fail to verify that the fuzzy system is stable, while Corollary 6 of this paper can obtain the better upper bounds than those in $[14,26]$. Moreover, it should be noted that $h_{d}=0$ does not mean a constant delay (i.e., $\dot{\tau}(t) \equiv 0$ ), but it means a time-varying delay with $\dot{\tau}(t) \leq 0, \forall t \geq 0$. Also, our result gives lesser conservativeness for a relatively large value or unknown case of $h_{d}$.

Furthermore, we will give another example to show the effectiveness and merit of nonlinear system via T-S fuzzy models, and the proposed approach also yields less conservative than the existing results. 
TABLE 4: MADB on $h_{b}$ for various $h_{d}\left(h_{a}=0\right)$.

\begin{tabular}{lcccc}
\hline Method & $h_{d}=0$ & $h_{d}=0.1$ & $h_{d}=0.5$ & $h_{d}$ unknown \\
\hline Chen et al. [25] & 3.150 & - & - & - \\
Liu et al. [26] & 3.300 & 2.650 & 1.500 & 0.790 \\
An et al. [14] & 3.704 & 3.011 & 1.657 & 1.197 \\
Corollary 6 $(\alpha=0.7)$ & 3.942 & 3.111 & 2.054 & 1.895 \\
Corollary 6 $(\alpha=0.8)$ & 4.062 & 3.288 & 2.076 & 1.779 \\
\hline
\end{tabular}

Example 3 (Example 1 of [9]). Consider the following timedelayed nonlinear system:

$$
\begin{aligned}
\dot{x}_{1}(t)= & 0.5\left(1-\sin ^{2}(\theta(t))\right) x_{2}(t)-x_{1}(t-\tau(t)) \\
& -\left(1+\sin ^{2}(\theta(t))\right) x_{1}(t), \\
\dot{x}_{2}(t)= & \operatorname{sgn}\left(|\theta(t)|-\frac{\pi}{2}\right)\left(0.9 \cos ^{2}(\theta(t))-1\right) x_{1}(t-\tau(t)) \\
& -x_{2}(t-\tau(t))-\left(0.9+0.1 \cos ^{2}(\theta(t))\right) x_{2}(t)
\end{aligned}
$$

which can be exactly expressed as a T-S delayed system (4) with the following rules $[8-10,13]$ :

$$
\begin{aligned}
& R^{1}: \text { if } \theta(t) \text { is } \pm \frac{\pi}{2}, \text { then } \\
& \dot{x}(t)=A_{1} x(t)+A_{\tau 1} x(t-\tau(t)), \\
& R^{2}: \text { if } \theta(t) \text { is } 0, \text { then } \\
& \dot{x}(t)=A_{2} x(t)+A_{\tau 2} x(t-\tau(t)) .
\end{aligned}
$$

The membership functions for above rules 1 and 2 are

$$
h_{1}\left(x_{1}(t)\right)=\sin ^{2}\left(x_{1}(t)\right), \quad h_{2}\left(x_{1}(t)\right)=\cos ^{2}\left(x_{1}(t)\right)
$$

with the following system parameters:

$$
\begin{array}{ll}
A_{1}=\left[\begin{array}{cc}
-2 & 0 \\
0 & -0.9
\end{array}\right], & A_{\tau 1}=\left[\begin{array}{cc}
-1 & 0 \\
-1 & -1
\end{array}\right], \\
A_{2}=\left[\begin{array}{cc}
-1 & 0.5 \\
0 & -1
\end{array}\right], & A_{\tau 2}=\left[\begin{array}{cc}
-1 & 0 \\
0.1 & -1
\end{array}\right] .
\end{array}
$$

To compare with the existing results, we assume that $h_{d}$ is unknown. The improvement of this paper is shown in Table 5. If the delay is fast time-varying case, the LMIs in Theorem 4 are feasible with $1.2 \leq \tau(t) \leq 1.634$. If the additional information $h_{d}=0.1$ is given, larger upper bounds of the delay can be computed by Theorem 4 , which is shown at the last row of Table 5.
TABLE 5: MADB on $h_{b}$ for varying $h_{a}$ ( $h_{d}$ unknown).

\begin{tabular}{lcccc}
\hline Method $\backslash h_{a}$ & 0.4 & 0.8 & 1.0 & 1.2 \\
\hline Lien et al. [6] & 0.8829 & 1.0677 & 1.1874 & 1.3181 \\
Peng et al. [8] & 1.0183 & 1.1817 & 1.2776 & 1.3816 \\
Li et al. [5] & 1.038 & 1.158 & 1.252 & 1.359 \\
Peng and Han [13] & 1.180 & 1.310 & 1.370 & 1.430 \\
Tian et al. [7] & 1.2647 & 1.3032 & 1.3528 & 1.4214 \\
An and Wen [12] & 1.277 & 1.311 & 1.358 & 1.419 \\
Peng and Fei [9] & 1.320 & 1.320 & 1.380 & 1.420 \\
Souza et al. [10] & 1.2836 & 1.3394 & 1.4009 & 1.4815 \\
Theorem $4(\alpha=0.7)$ & 1.537 & 1.556 & 1.586 & 1.634 \\
Theorem $4\left(\alpha=0.7\right.$ and $\left.h_{d}=0.1\right)$ & 2.079 & 2.058 & 2.029 & 1.986 \\
\hline
\end{tabular}

As an example, when $h_{a}=1.2, h_{b}=1.634$, and $h_{d}$ is unknown, by applying Theorem 4 in this paper, we can obtain the following solutions:

$$
\begin{gathered}
P=\left[\begin{array}{ll}
0.2142 & 0.0715 \\
0.0715 & 0.0842
\end{array}\right], \quad S_{11}=\left[\begin{array}{ll}
0.1455 & 0.0346 \\
0.0346 & 0.0330
\end{array}\right], \\
S_{12}=\left[\begin{array}{ll}
-0.0090 & -0.0220 \\
-0.0084 & -0.0274
\end{array}\right], \quad S_{22}=\left[\begin{array}{ll}
0.1534 & 0.0460 \\
0.0460 & 0.0641
\end{array}\right], \\
R_{1}=\left[\begin{array}{ll}
0.0475 & 0.0486 \\
0.0486 & 0.0822
\end{array}\right], \quad R_{2}=\left[\begin{array}{ll}
0.0317 & 0.0217 \\
0.0217 & 0.0305
\end{array}\right], \\
R_{3}=\left[\begin{array}{ll}
0.4544 & 0.1034 \\
0.1034 & 0.1084
\end{array}\right], \quad R_{4}=\left[\begin{array}{ll}
0.0779 & 0.0365 \\
0.0365 & 0.0421
\end{array}\right], \\
Q_{1}=\left[\begin{array}{ll}
0.1375 & 0.0246 \\
0.0246 & 0.0202
\end{array}\right], \quad Q_{2}=\left[\begin{array}{ll}
0.1513 & 0.0390 \\
0.0390 & 0.0409
\end{array}\right], \\
Q_{3}=\left[\begin{array}{cc}
0.1738 & 0.0541 \\
0.0541 & 0.0345
\end{array}\right]
\end{gathered}
$$

Furthermore, with initial state condition $\phi(t)=[1,-1]^{T}$, Figure 1 depicts the state responses of the system (41) with $1.2 \leq \tau(t) \leq 1.634$ listed in Table 5 .

\section{Conclusion}

Two delay-interval-dependent stability criteria have been proposed for T-S fuzzy systems with interval time-varying delays through constructing a novel delay-dependent LK functional via variable delay partitioned approach, utilizing suitably some integral inequalities, and employing convex combination technique and a scalar inequality. The proposed criteria provide better results since approximation of the uncertain delay factors in the time-derivative of LK functional has been avoided, while the positiveness of LK functional also must not be guaranteed by imposing the positiveness of all the terms composing the LK functional separately. The superiority of the present result has been validated through numerical examples. Moreover, the proposed 


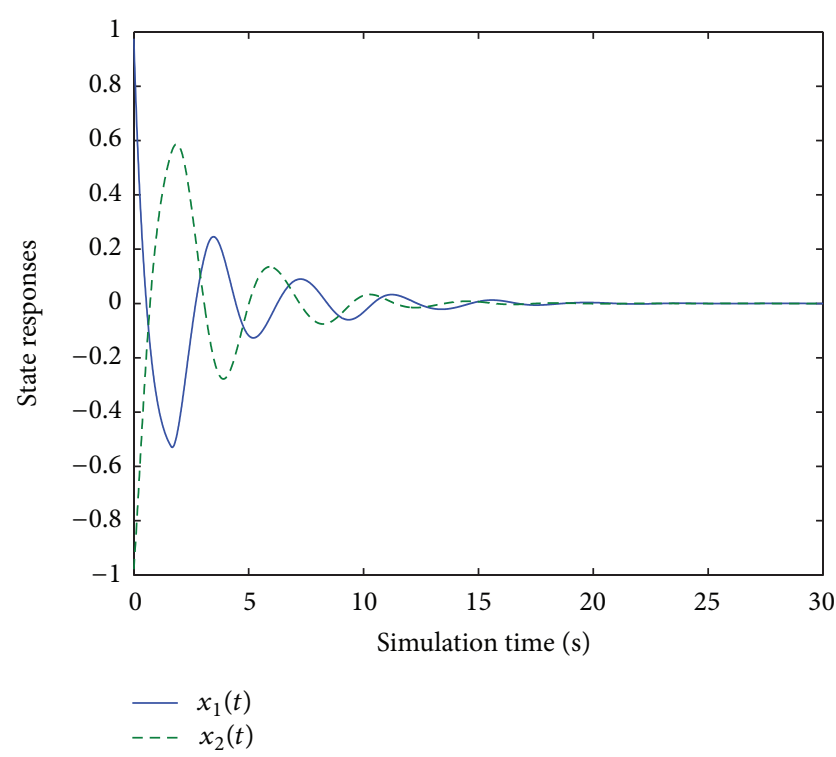

FIGURE 1: State responses of the system (41) and (42) for Example 3.

approach is simple and may easily be extended to robust stabilization and $H_{\infty}$ control problems for uncertain timedelayed T-S fuzzy systems.

\section{Conflict of Interests}

The authors declare that there is no conflict of interests regarding the publication of this paper.

\section{Acknowledgments}

This work is supported in part by the Natural Science Foundation of China under Grant nos. 61173036 and 61370097, in part by the State Scholarship Fund of China Scholarship Council (CSC) under Grant no. 201206135001, and in part by the Planned Science and Technology Project of Hunan Province under Grant no. 2012FJ4130.

\section{References}

[1] K. Gu, V. L. Kharitonov, and J. Chen, Stability of Time-Delay Systems, Birkhäuser, Basel, Switzerland, 2003.

[2] T. Takagi and M. Sugeno, "Fuzzy identification of systems and its applications to modeling and control," IEEE Transactions on Systems, Man and Cybernetics, vol. 15, no. 1, pp. 116-132, 1985.

[3] Y.-Y. Cao and P. M. Frank, "Stability analysis and synthesis of nonlinear time-delay systems via linear Takagi-Sugeno fuzzy models," Fuzzy Sets and Systems, vol. 124, no. 2, pp. 213-229, 2001.

[4] K. Tanaka and H. O. Wang, Fuzzy Control Systems Design and Analysis: A Linear Matrix Inequality Approach, John Wiley \& Sons, New York, NY, USA, 2001.

[5] L. Li, X. Liu, and T. Chai, "New approaches on $H_{\infty}$ control of T-S fuzzy systems with interval time-varying delay," Fuzzy Sets and Systems, vol. 160, no. 12, pp. 1669-1688, 2009.
[6] C. H. Lien, K. W. Yu, W. D. Chen, Z. L. Wan, and Y. J. Chung, "Stability criteria for uncertain Takagi-Sugeno fuzzy systems with interval time-varying delay," IET Control Theory \& Applications, vol. 1, no. 3, pp. 764-769, 2007.

[7] E. Tian, D. Yue, and Y. Zhang, "Delay-dependent robust $H_{\infty}$ control for T-S fuzzy system with interval time-varying delay," Fuzzy Sets and Systems, vol. 160, no. 12, pp. 1708-1719, 2009.

[8] C. Peng, L.-Y. Wen, and J.-Q. Yang, "On delay-dependent robust stability criteria for uncertain T-S fuzzy systems with interval time-varying delay," International Journal of Fuzzy Systems, vol. 13, no. 1, pp. 35-44, 2011.

[9] C. Peng and M.-R. Fei, "An improved result on the stability of uncertain T-S fuzzy systems with interval time-varying delay," Fuzzy Sets and Systems, vol. 212, pp. 97-109, 2013.

[10] F. O. Souza, V. C. S. Campos, and R. M. Palhares, "On delay-dependent stability conditions for Takagi-Sugeno fuzzy systems," Journal of the Franklin Institute. In press.

[11] F. O. Souza, L. A. Mozelli, and R. M. Palhares, "On stability and stabilization of T-S fuzzy time-delayed systems," IEEE Transactions on Fuzzy Systems, vol. 17, no. 6, pp. 1450-1455, 2009.

[12] J. Y. An and G. L. Wen, "Improved stability criteria for time-varying delayed T-S fuzzy systems via delay partitioning approach," Fuzzy Sets and Systems, vol. 185, no. 1, pp. 83-94, 2011.

[13] C. Peng and Q.-L. Han, "Delay-range-dependent robust stabilization for uncertain T-S fuzzy control systems with interval time-varying delays," Information Sciences, vol. 181, no. 19, pp. 4287-4299, 2011

[14] J. Y. An, T. Li, G. L. Wen, and R. F. Li, "New stability conditions for uncertain T-S Fuzzy systems with interval timevarying delay," International Journal of Control, Automation and Systems, vol. 10, no. 3, pp. 490-497, 2012.

[15] M. Chadli and T. M. Guerra, "LMI solution for robust static output feedback control of Takagi-Sugeno fuzzy models," IEEE Transactions on Fuzzy Systems, vol. 20, no. 6, pp. 1160-1165, 2012.

[16] X.-L. Zhu and G.-H. Yang, "Jensen integral inequality approach to stability analysis of continuous-time systems with timevarying delay," IET Control Theory and Applications, vol. 2, no. 6, pp. 524-534, 2008.

[17] L. A. Mozelli, R. M. Palhares, F. O. Souza, and E. M. A. M. Mendes, "Reducing conservativeness in recent stability conditions of TS fuzzy systems," Automatica, vol. 45, no. 6, pp. $1580-1583,2009$.

[18] Z. Yang and Y.-P. Yang, "New delay-dependent stability analysis and synthesis of T-S fuzzy systems with time-varying delay," International Journal of Robust and Nonlinear Control, vol. 20, no. 3, pp. 313-322, 2010.

[19] M. Chadli, A. Abdo, and S. X. Ding, " $H_{-} / H_{\infty}$ fault detection filter design for discrete-time Takagi-Sugeno fuzzy system," Automatica, vol. 49, no. 7, pp. 1996-2005, 2013.

[20] D. Saifia, M. Chadli, S. Labiod, and H. R. Karimi, " $H_{\infty}$ fuzzy control of DC-DC converters with input constraint," Mathematical Problems in Engineering, vol. 2012, Article ID 973082, 18 pages, 2012.

[21] S. Aouaouda, M. Chadli, V. Cocquempot, and M. Tarek Khadir, "Multi-objective $H_{-} / H_{\infty}$ faults detection observer design for Takagi-Sugeno fuzzy systems with unmeasurable premise variables: descriptor approach," International Journal of Adaptive Control and Signal Processing, vol. 27, no. 12, pp. 1031-1047, 2012.

[22] S. Aouaouda, M. Chadli, M. Tarek Khadir, and T. Bouarar, "Robust fault tolerant tracking controller design for unknown 
inputs T-S models with unmeasurable premise variables," Journal of Process Control, vol. 22, no. 5, pp. 861-872, 2012.

[23] M. Chadli and H. R. Karimi, "Robust observer design for unknown inputs Takagi-Sugeno models," IEEE Transactions on Fuzzy Systems, vol. 21, no. 1, pp. 158-164, 2013.

[24] X.-M. Zhang, M. Wu, J.-H. She, and Y. He, "Delay-dependent stabilization of linear systems with time-varying state and input delays," Automatica, vol. 41, no. 8, pp. 1405-1412, 2005.

[25] B. Chen, X. Liu, and S. Tong, "New delay-dependent stabilization conditions of T-S fuzzy systems with constant delay," Fuzzy Sets and Systems, vol. 158, no. 20, pp. 2209-2224, 2007.

[26] F. Liu, M. Wu, Y. He, and R. Yokoyama, "New delay-dependent stability criteria for T-S fuzzy systems with time-varying delay," Fuzzy Sets and Systems, vol. 161, no. 15, pp. 2033-2042, 2010.

[27] Q.-L. Han and K. Gu, "Stability of linear systems with timevarying delay: a generalized discretized lyapunov functional approach," Asian Journal of Control, vol. 3, no. 3, pp. 170-180, 2001.

[28] P.-L. Liu, "Delay-range-dependent stability criteria for TakagiSugeno fuzzy systems with fast time-varying delays," Journal of Applied Mathematics, vol. 2012, Article ID 475728, 20 pages, 2012.

[29] Y. Zhao, H. Gao, J. Lam, and B. Du, "Stability and stabilization of delayed T-S fuzzy systems: a delay partitioning approach," IEEE Transactions on Fuzzy Systems, vol. 17, no. 4, pp. 750-762, 2009.

[30] J. Sun, G. P. Liu, J. Chen, and D. Rees, "Improved delay-rangedependent stability criteria for linear systems with time-varying delays," Automatica, vol. 46, no. 2, pp. 466-470, 2010.

[31] C. Briat, "Convergence and equivalence results for the Jensen's inequality-application to time-delay and sampled-data systems," IEEE Transactions on Automatic Control, vol. 56, no. 7, pp. 1660-1665, 2011.

[32] Y. Wu, Y. Wu, and Y. Chen, "Mean square exponential stability of uncertain stochastic neural networks with time-varying delay," Neurocomputing, vol. 72, no. 10-12, pp. 2379-2384, 2009. 


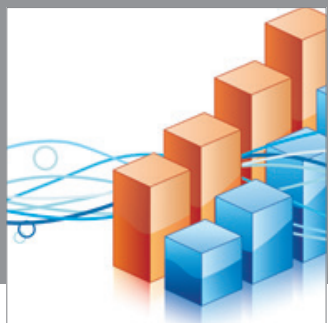

Advances in

Operations Research

mansans

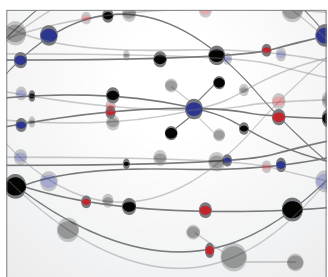

The Scientific World Journal
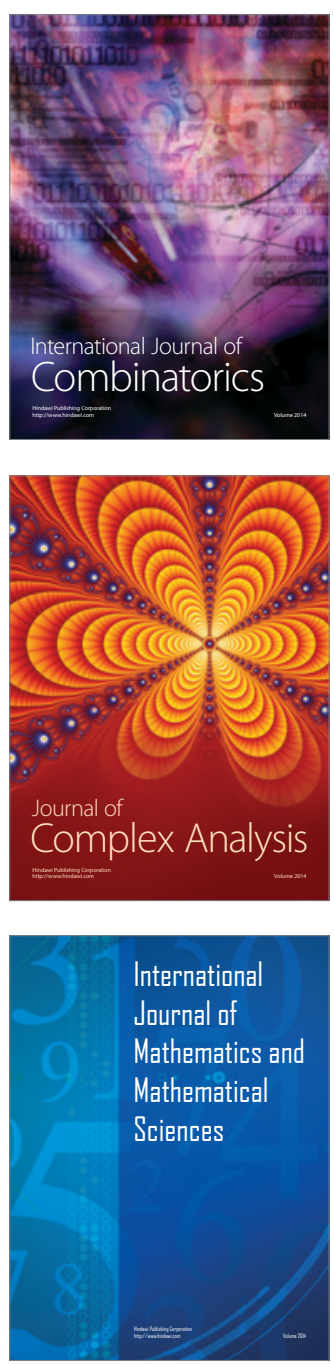
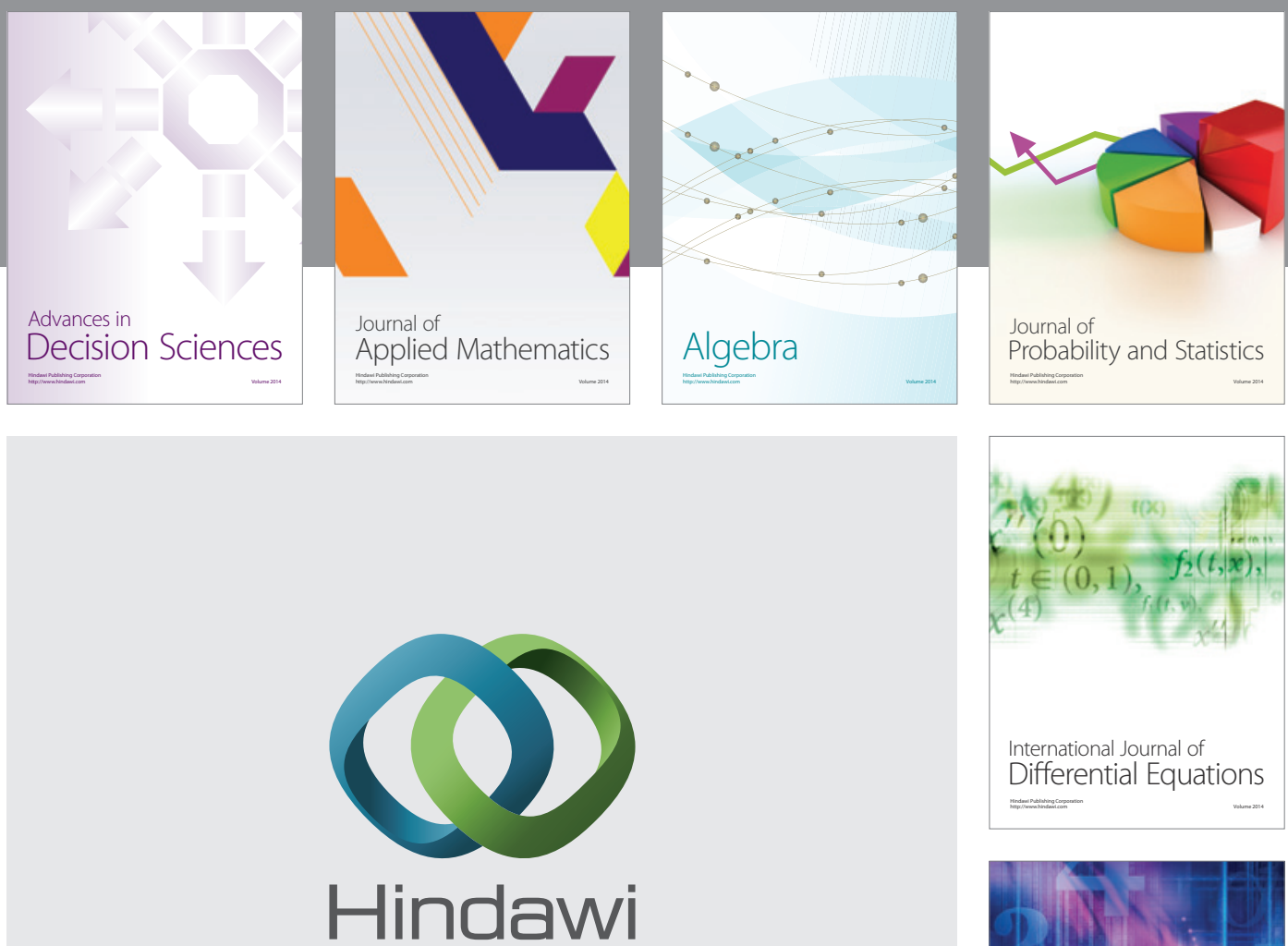

Submit your manuscripts at http://www.hindawi.com
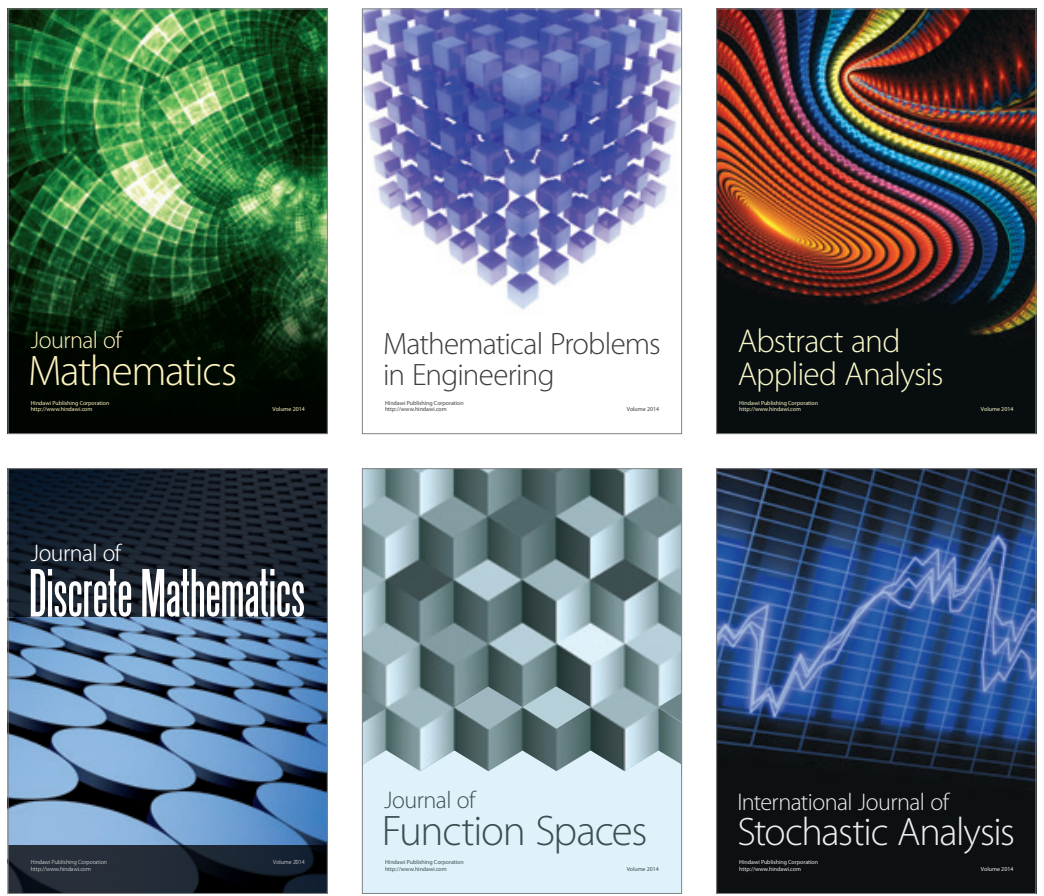

Journal of

Function Spaces

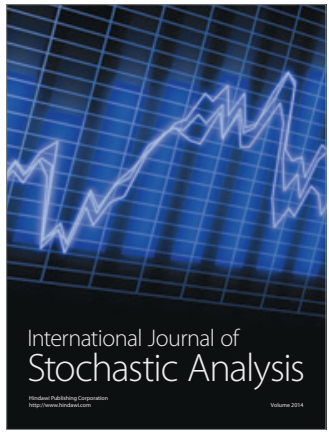

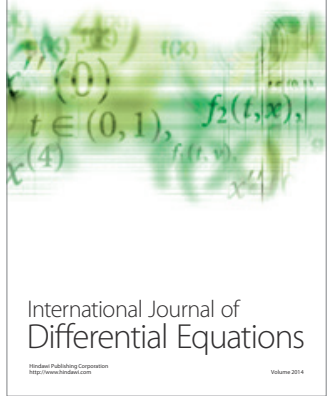
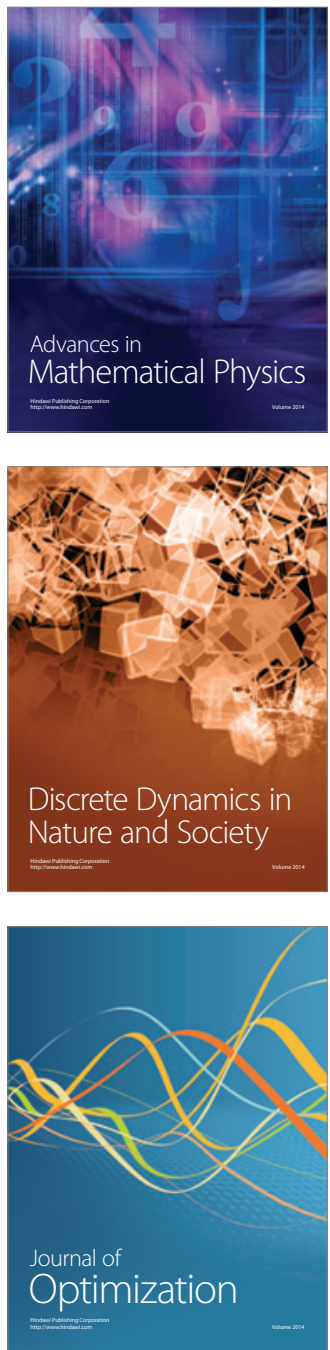